\title{
American Experience with Extracorporeal Support in Covid-19 Patients: Early Outcomes from a Single Institution
}

\author{
Erik Osborn ${ }^{1}$, Alan Speir ${ }^{1}$, James Lantry ${ }^{1}$, Chris King ${ }^{1}$, Ramesh Singh ${ }^{1}$, Liam Ryan ${ }^{1}$, Jikar \\ Simou ${ }^{1}$, Eric Sarin ${ }^{1}$, Daniel Tang ${ }^{1}$, Sarah Hatch ${ }^{1}$, Mary Looby ${ }^{1}$, Joby Chandy ${ }^{1}$, Jeremy \\ Gold $^{1}$, Laith Altaweel ${ }^{1}$, Kevin Lowery ${ }^{1}$, Manoj Reddy ${ }^{1}$, Jing Wang ${ }^{1}$, Kathleen Petro ${ }^{1}$, \\ Heidi Dalton ${ }^{1}$, Hussain Dhanani ${ }^{1}$, Patrick Moran ${ }^{1}$, Svetolik Djurkovic ${ }^{1}$, Pouya \\ Tahsilifahadan $^{1}$, Thomas Preston ${ }^{1}$, and Mehul Desai ${ }^{1}$ \\ ${ }^{1}$ Inova Health System
}

July 1, 2020

\begin{abstract}
As the world learned about Covid-19, the application of ECMO also evolved as health systems in the United States had some time to prepare. We report our initial experience using extracorporeal support for Covid-19 patients with the resource challenges that attend a worldwide pandemic.
\end{abstract}

\section{Background:}

Extracorporeal support has improved survival in select neonatal and pediatric patients for over forty years. Over the last two decades, extracorporeal membrane oxygenation (ECMO) has emerged as an acceptable and potentially beneficial rescue modality in select adult populations. In severe respiratory failure, ECMO provides time for the lungs to rest and recover by augmenting gas exchange with the extracorporeal circuit.

A review of the outcomes in patients with Middle East Respiratory Syndrome Coronavirus (MERS-CoV) treated with ECMO demonstrated a decrease in hospital mortality rate and length of intensive care unit (ICU) stay compared to those managed with conventional therapy alone (1). Early reports regarding the use of ECMO in patients with Severe Acute Respiratory Distress Syndrome Coronavirus 2 (SARS-COV-2 or Covid-19) pneumonia have been mixed. Despite the similarities of MERS-CoV and the current novel coronavirus disease, recent reports have raised concerns regarding the high mortality rates observed in an early series of ECMO supported Covid-19 patients $(2,3)$. Similarly, a pooled analysis of five recent studies $(4,5,6,7,8)$ suggested ECMO produced neither harm nor benefit in Covid-19 patients with ARDS. The authors of these analysis compared mortality in patients supported with ECMO for both MERS and Covid19 and concluded Covid-19 patients had a significantly higher mortality than MERS (94.1\% vs. $65.0 \%)$ when treated with ECMO. The authors further state that this data raises "questions about the real utility of ECMO in this outbreak" (8). Initial reports from United States hospital systems that had been both surprised and overrun with critically ill Covid-19 patients, demonstrated unfavorable results with supporting patients with ECMO, leading centers to abandon this support modality for these patients (9).

We present a single-center analysis and preliminary outcomes regarding the use of ECMO in 15 patients with severe respiratory failure due to Covid-19 all of which had failed maximal conventional ventilatory management and interventions as presented. These initial promising results prompted communication of our experience, despite its preliminary nature.

Methods : 
All patients placed on ECMO due to COVID-19 pneumonia from March $10^{\text {th }}, 2020$ to April $30^{\text {th }}, 2020$ were identified. Data was manually extracted from the medical records of all fifteen patients into the ECMO registry. All patients were admitted to either the medical surgical intensive care unit (MSICU) or cardiovascular intensive care unit (CVICU) between March 15 th, 2020 , and April 27 $7^{\text {th }}, 2020$. This study was approved by the Institutional review board.

Patients were managed by the ECMO team, which consisted of an experienced ECMO physician, cardiac surgeon, ECMO nurse, ECMO specialist and respiratory therapist. Prior to the Covid-19 pandemic, ECMO patients had only been cared for in the CVICU. Do to overwhelming number and severity of the respiratory failure, extension into the MSICU was necessary. Placement and care of ECMO patients within the MSICU was a new practice and required sharing of resources from the CVICU and included CVICU ECMO nurses, physicians and ECMO specialists to care for the ECMO patients. The CVICU nurse were additionally tasked with training the MSICU nurses.

\section{Patient Selection for Extracorporeal Support -}

Extensive discussions with a multidisciplinary team composed of ECMO physicians, cardiac surgeons, medical intensivists, and senior ECMO providers led to more restrictive criteria than is usually utilized for respiratory ECMO patient selection. The experiences in Asia, Europe and early United States were considered while preparing for this global pandemic.

Specific criteria for patients to be considered and accepted for VV ECMO include (Figure 1):

A) Ventilated less than 10 days with no additional serious comorbidities

B) Patients less than 60 years of age

C) $\mathrm{PaO}_{2} / \mathrm{FiO}_{2}$ ratio $<100$ and/or patients that were not adequately supported despite:

1. Lung protective ventilation:

(Tidal Volume $(\mathrm{Vt})<6 \mathrm{ml} / \mathrm{kg}$ Ideal body weight (IBW), Plateau Pressure (PPlat) $<30 \mathrm{mmHg} *$ )

2. Neuromuscular blockade (NMB)

3. Prone positioning

D) Hypercapnic acidosis compromising perfusion with failed above interventions

The term "serious comorbidities" refers to the fact that patients with chronic organ dysfunction due to co-morbidities outside of diabetes mellitus (DM), hypertension (HTN), and hyperlipidemia (HLD) were excluded. As indicated, an age less than 60 years was initially utilized as the upper limit for ECMO candidacy. This age limit was further reduced to patients $<55$ years as ECMO resources became limited. If the $\mathrm{P} / \mathrm{F}$ ratio was $>100$ and lung protective ventilation was not providing adequate gas exchange (compatible with perfusing vital organs), the patient was considered for ECMO. For the purposes of defining the above criteria: Failure of adequate gas exchange was defined as an oxygen saturation less than $85 \%, \mathrm{PaO}_{2}<50 \mathrm{mmHg}$, or an acidosis compromising perfusion ( $\mathrm{pH}<7.2$ with hypercarbia) for more than 3 hours. Perfusion compromise was defined as a mean arterial pressure less than $65 \mathrm{mmHg}$. Failure of lung protective ventilation is defined as the requirement of a PPlat $>30 \mathrm{mmHg}$ and/or a Vt $>6 \mathrm{ml} / \mathrm{kg}$ IBW for more than 3 hours to achieve adequate gas exchange. Pulmonary vasodilator therapy with inhaled epoprostenol or inhaled nitric oxide were used as adjunctive measures in addition to lung protective ventilation.

Covid-19 patients with concomitant hemodynamic instability due to a reversible cause were evaluated for Veno-Arterial (VA)-ECMO on a case by case basis. An example of a potentially reversible cause would be acute right heart failure. When VA-ECMO is initiated, the family is informed that 5-7 days of support will be provided. If there is no improvement after this allotted time, goals of care and discontinuation of ECMO will be discussed. The ECMO team decided that extracorporeal CPR (eCPR) will not be offered for this patient population. 
The decision to place patients on ECMO was made by a multidisciplinary team approach that included an experienced ECMO physician, cardiac surgeon, and intensivist. For patients that were borderline or at higher risk, additional ECMO intensivists and cardiac surgeons were consulted. In anticipation of limited resources, ECMO was deployed for patients with the perceived highest chance of survival based on their age and prior level of health.

\section{Pre-ECMO Management-}

All patients excluding outside transfers were admitted and managed with lung protective ventilation, NMB, and prone positioning. Twelve of the fifteen patients were managed with lung protective ventilation prior to initiation of ECMO. The three patients that were not treated with lung protective ventilation were patients transferred to our institution from outside hospitals. Of the three transfers, one patient expired peri-cannulation, one patient was decannulated after 17 days and remains on MV, and one patient was successfully decannulated on ECMO day 16 and has been discharged to home. Patients supported on mechanical ventilation without lung protective ventilation remained on ECMO longer than those that only received lung protective ventilation. Neuromuscular blockade was utilized in 14 of 15 patients. The NMB exception was a patient transferred from an outside hospital who expired immediately after cannulation. 14 of 15 patients were placed in the prone position. The exception to prone positioning was due to body habitus limitations (BMI 44). Two patients did not receive inhaled vasodilator therapy prior to ECMO cannulation; seven received inhaled epoprostenol and six received inhaled nitric oxide. From onset of Covid-19 symptoms to initiation of ECMO, the average time was 14 days, with a range of $4-29$ days. The average duration of mechanical ventilation (MV) prior to ECMO was 4.5 days, with a range of 1-9 days. As expected, all patients had elevated inflammatory markers (d-dimer, CRP, ferritin). The Pre-ECMO characteristics of the patients are listed in Tables $1 \& 2$.

\section{Cannulation and ECMO initiation-}

Femoral-femoral veno-venous ECMO cannulation was performed by the cardiac surgeon in the ICU under trans-esophageal (TEE) guidance by cardiac anesthesia. Full personal protective equipment was worn by all members of the health care team within the room. Additional precautionary measures included limiting the number of health care workers within the patients' room during cannulation. The institutional cannulation heparin bolus dose for VV ECMO was increased from $50 \mathrm{u} / \mathrm{kg}$ to $100 \mathrm{u} / \mathrm{kg}$ or more after the initial patients formed significant thromboses despite heparin anticoagulation. Cannula included a Edwards ThruPort Quick Draw (Irvine, CA) 25Fr multi-stage femoral venous drain with the distal tip positioned at approximately the hepatic vein - inferior vena cava junction, and a Medtronic Bio-Medicus NextGen (Minneapolis, MN) 19-21Fr return cannula with the tip placed within the right atrium. ECMO equipment consisted of either a Rotaflow centrifugal pump (Getinge AB, Getinge, Sweden) with a Quadrox iD Adult oxygenator or a CARDIOHELPi system (Getinge AB) with HLS Set Advanced 7.0. ECMO blood flow was maintained $>3$ $\mathrm{L} / \mathrm{min}$ to help minimize the identified risk of thrombosis.

Following cannulation, patients were placed on an ultra-protective ventilator strategy that included a graded reduction in plateau pressure to $<20 \mathrm{cmH}_{2} \mathrm{O}$ over the next 12 hours. Tidal volumes were reduced to $<$ $4 \mathrm{ml} / \mathrm{kg}$ IBW and the $\mathrm{FiO}_{2}$ was reduced to $60 \%$ if the patient was able to maintain an acceptable oxygenation status. Positive end expiratory pressure (PEEP) was slowly reduced to a level of $10-14 \mathrm{cmH}_{2} \mathrm{O}$. Higher levels of PEEP were required in patients with high BMI's. Respiratory rate was maintained at $8-10$ breaths/minute for the initial 24 hours. In Covid-19 patients with the lung phenotype characterized with increased compliance, higher tidal volumes, and respiratory rates were tolerated as the patients started to improve (10). In addition, patients with bilateral infiltrative disease that did not improve after fluid removal and lung rest underwent a 16-hour prone positioning plan (Figure 2). Seven of the fifteen patients managed on ECMO were placed into a prone position at least once, with the majority in prone position multiple times. Mobility was gradually enhanced from a bed tilt of 30-45 degrees to as high as standing (vertical) positioning via a specialty bed (Kreg Medical, Inc. Chicago, IL) (Figure 3).

\section{ECMO Course-}


After one week of ECMO support patients were evaluated regarding their potential need for tracheostomy placement based on their clinical progress. Patients whose lung function showed little improvement or who displayed significant agitation or ventilator dysynchrony during sedation holidays were considered for tracheostomy placement. A tracheostomy was performed in 6 of the Covid-19 ECMO patients. Anticoagulation was continued and the tracheostomy was placed utilizing a percutaneous approach with electrocautery to minimize bleeding. The number of providers in the room was again limited to the ECMO physician/intensivist, bedside RN, cardiac surgeon, and an operating room assistant. During placement of the tracheostomy, the ventilator was placed in standby mode. Two patients had minor bleeding which was controlled through electrocautery without interruption of anticoagulation.

\section{Anticoagulation and thrombosis-}

Considering the known Covid-19 associated thrombotic microangiopathy and early pro-thrombotic tendencies of the other Covid-19 patients, the normal low intensity heparin protocol was prophylactically increased to a moderate intensity heparin protocol (11). At our institution, an in-vivo therapeutic range of heparin (unfractionated anti-Xa range of $0.3-0.5 \mathrm{IU} / \mathrm{mL}$ ) correlates with an aPTT range of $64-102$ seconds. Given the bleeding and thrombotic complications in ECMO patients, a tighter range of $70-90$ seconds was targeted post-cannulation. If a patient demonstrated a poor response to heparin, defined by as significant clot formation during cannulation, increasing fibrin deposition on the inlet or outlet of the oxygenator, frequent clotting within the CRRT circuit, or type 2 heparin induced thrombocytopenia (HIT) despite an aPTT within range, systemic anticoagulation was transitioned to bivalirudin with a goal aPTT of 75-95 seconds. Eleven patients were started on a heparin infusion post-cannulation while three patients were started on a bivalirudin infusion. Of the 11 patients initially on the heparin infusion, 8 were later transitioned to bivalirudin due to the reasons cited above. Additional details on the anticoagulation course are shown in Table 3. During the transition from heparin to bivalirudin, the bivalirudin was infused for 30 minutes prior to heparin discontinuation. Aspirin was administered on a case by case basis dependent upon clot formation within the circuit as well as in cases with evidence of an elevated maximum amplitude (MA) on the thromboelastograph (TEG). A transfusion policy was maintained for packed red blood cells, with administration for a hemoglobin $<8 \mathrm{~g} / \mathrm{dL}$ with evidence of inadequate oxygen delivery. Platelets were maintained $>50,000 / \mathrm{mm}^{3}$ unless the patient was actively bleeding.

\section{Anticoagulation after ECMO Decannulation-}

After decannulation, patients were maintained on intravenous heparin or bivalirudin for four days. The PTT goal was lowered one day after decannulation to 50-70 seconds. Ultrasound studies were performed on patients two to three days post decannulation and deep venous thrombosis was identified in 4 patients (femoral veins and internal jugular vein). The d-dimer remained elevated in all patients. Once discharged from the ICU, the patients were transitioned to either a treatment dose enoxaparin $1 \mathrm{mg} / \mathrm{kg}$ or oral apixaban $5 \mathrm{mg}$ twice daily. This empiric anticoagulation in the setting of elevated d-dimer testing was in response to the high rate of pulmonary embolism (PE) reported in multiple case series. For example, a 25\% PE rate was reported in a multicenter study from France of 150 patients (12). The patients were discharged on oral apixaban with hematology follow up scheduled in two weeks to recheck their d-dimer and determine the length of apixaban treatment.

\section{Continuous Renal Replacement Therapy-}

Most ECMO patients were also rapidly started on continuous renal replacement therapy (CRRT) for precise volume management and the potential added benefit of cytokine removal. High flow pre-filter replacement fluid was maintained at 6 liters to remove cytokines through convective clearance. (Table 4) Maintaining a replacement fluid rate at 6 liters required significant nursing time. Attempting to pull CRRT from the ECMO circuit post oxygenator and return to the ECMO circuit post pump/pre-oxygenator created an additional challenge of maintaining high CRRT blood flowrates due to the CRRT return pressure. This was remedied by returning the CRRT circuit blood directly to the venous drainage line (pre-pump) of the ECMO circuit (Figure 4). High flow CRRT was performed under an investigational protocol, as there is a dearth of good 
evidence that cytokine removal impacts outcomes (13). Due to the prothrombotic nature of the Covid-19 patients, regional citrate anticoagulation was utilized for the CRRT circuits even though all patients were concurrently systemically anticoagulated.

Patients were placed on a regional citrate protocol unless they had evidence of hepatic dysfunction. If the CRRT circuit clotted repeatedly despite therapeutic levels of heparin and citrate, patients were transitioned to the bivalirudin anticoagulation strategy. The indications for CRRT were volume removal or acute kidney injury, and due to the limited supply of replacement fluid, the duration of high flow clearance was truncated for many patients. The levels of inflammatory markers predictably decreased, and CRRT was discontinued in patients with recovering renal function once the excess fluid had been removed or adequate fluid removal was achievable with loop diuretic infusions.

\section{Complications-}

Thrombotic complications were the most prevalent problem and were identified in $66 \%$ of the patients. Thrombotic complications were defined as excessive clotting during cannulation (clotted cannula despite pre-cannulation heparin bolus), early excessive fibrin formation in the oxygenator leading to a decreased post-oxygenator $\mathrm{PaO}_{2}$, repeated CRRT filter clot (in the presence of therapeutic anticoagulation), visible ECMO circuit thrombosis requiring emergent circuit change, pulmonary embolus (PE), type 2 HIT, and post ECMO deep venous thrombosis. Secondary infections occurred in seven of the fifteen patients and were treated effectively with broad spectrum antibiotics. The most common secondary infection was pneumonia. One patient developed a multidrug resistant Pseudomonas pneumonia infection after ECMO decannulation from ECMO. Two patients developed a pneumothorax after decannulation that required small bore chest tube placement.

Gastrointestinal (GI) bleeding requiring massive transfusion occurred in two patients. In one patient the GI bleed occurred after a tissue plasminogen activator (tPA) infusion while receiving a concurrent heparin infusion followed by a tPA/bivalirudin infusion. The tPA infusion was attempted to treat a presumed pulmonary micro-thrombosis in a patient with right ventricular (RV) failure after a PE that had caused a cardiac arrest. His cardiac arrest responded quickly to 50mg of tPA infusion and $1 \mathrm{mg}$ of epinephrine. The tPA infusion improved his RV failure minimally, but not significantly enough to avoid a period of VVA ECMO (14). This patient required VVA ECMO after an initial 21 days of VV ECMO. He was successfully decannulated after 5 days of VVA ECMO. In addition to the patient with the large PE, two other patients developed RV failure. One required VVAV ECMO cannulation, with drainage from the femoral vein and superior vena cava and return to the femoral artery and right atrium. The third patient with RV failure also required VAV support along with milrinone and an epinephrine infusion.

\section{Neurologic Complications and Manifestations-}

SARS-CoV-2 virus enters the nervous system by hematogenous spread or retrograde neuronal transmission via the olfactory bulb and enters neuronal and glial cells by attaching to the angiotensin-converting enzyme 2 (ACE2) receptor, widely expressed in various neuronal populations (15). A wide range of neurologic manifestations have been reported in more than a third of the Covid-19 patients, particularly those with a severe infection (16). In addition, neurologic complications, particularly cerebrovascular diseases, represent a major cause of morbidity and mortality for ECMO patients $(17,18)$.

All Covid-19 patients on ECMO support underwent routine neurological examination. Sixty percent of the ECMO patients developed significant neurologic complications affecting both central and peripheral nervous systems. The most common neurological complications were encephalopathy, seizures, autonomic dysfunction, and critical illness polyneuropathy (Table 5).

Encephalopathy, defined broadly as an alteration in mental status, was seen in 6 patients and can be attributed to a variety of etiologies. All 9 extubated patients can follow commands and show gradual improvement in mental status. Six patients underwent continuous electroencephalography (EEG) monitoring using leads placed into the subgaleal space. Neurologists with advanced EEG training reviewed both raw 
and quantitative EEG data in correlation with patients' neurologic exam and hemodynamic parameters. All six patients showed abnormal epileptiform activity. (Figure 5) In three patients, the epileptiform activity correlated with a change in neurologic exam, and both EEG findings and neurologic exams improved after the administration of antiseizure medication. Two patients, who remain on ECMO support at the time of this report, had epileptiform activity without a clinical correlate that also resolved with antiseizure medication. Seven patients developed wide fluctuations of heart rate and blood pressure out of proportion to known stimuli that was suggestive of autonomic dysfunction. Four of these patients had poorly controlled DM rendering it difficult discern between diabetic autonomic neuropathy versus other etiology. We did not find any clinical or radiographic evidence of brain ischemia or hemorrhage in our patients. Transcranial Doppler, obtained in one patient, did not reveal any high-intensity signal transients suggestive of cerebral micro emboli. Brain MRI obtained in two of the patients with seizure activity did not show any acute intracranial finding.

Six patients developed generalized weakness concerning for critical illness myopathy and or polyneuropathy; however, confirmatory nerve conduction and electromyographic studies were not obtained to limit provider exposure. The four patients who have been discharged from the hospital have shown continued improvement in their muscle strength. As this is a preliminary report, the patients' functional neurologic outcome remains in evolution. Cerebral Performance Category (CPC) will be obtained during follow up visits after discharge.

The underlying mechanisms of neurological complications in Covid-19 patients is still unclear and may involve both direct and indirect effects of SARS-CoV-2 infection as part of the systemic inflammatory response. Coagulopathy, myocardial injury, and pre-morbid risk factors, such as hypertension and diabetes, may also contribute to the development of neurological complications in these patients.

\section{Results and Discussion:}

Prior reports of ECMO use in Covid-19 patients with severe ARDS may need to be considered in the context of an unprecedented pandemic. Our experience demonstrates that ECMO can be successfully employed as a support modality for Covid-19 related ARDS in an appropriately resourced facility with judicious candidate selection. Health systems that were overwhelmed by Covid-19 patients were forced to abandon their normal practices in the presence of such unanticipated clinical demands. Standards of care change, like mass casualty events in the combat theater, as resources and staff are critically stretched. Many of these reports come from health systems operating at surge capacity (19). The case series described above demonstrates that extracorporeal support has had a beneficial role in carefully selected patients.

Of the fifteen Covid-19 ECMO patients, 11 have been successfully liberated from ECMO (Figure 6). Of the eleven that have been decannulated, 9 have been liberated from mechanical ventilation. At the time this article was written, all 11 of the patients removed from ECMO are following commands and have a chance at a good overall outcome (CPC score of 1-2) (Table 6) (20). Of the 9 patients liberated from MV, 4 have been sent home and 5 are recovering on the wards. Two of the patients still in the hospital are recovering from encephalopathy and critical illness polyneuropathy (Figure 7).

The potentially promising outcomes of the Covid-19 ECMO patients exist due to a multiplicity of reasons. The experiences of our colleagues around the world helped systems such as ours prepare for the Covid-19 pandemic and restrict selection criteria for the application of extracorporeal support. By selecting younger healthier patients, limited resources were used on patients more likely to survive with a good outcome. Instead of focusing on each individual as they presented, the focus moved to the collective group (21). Judicious use of ECMO may improve survival in carefully selected candidates (22).

Pre ECMO ventilator management that served to protect the lungs from additional injury may have been another supportive factor in the outcomes. A lung protective strategy of lower volumes and lower plateau pressures was maintained for most of our patients' pre ECMO ventilator time. As soon as lung protection failed, namely plateau pressures higher than $30 \mathrm{cmH}_{2} \mathrm{O}, \mathrm{ECMO}$ was initiated, thereby minimizing the period of additional lung injury (23). The patients whose lungs experienced higher pressures and volumes prior to ECMO had longer periods of ECMO support. Most patients also received NMB and trials of prone 
positioning prior to ECMO.

The higher levels of anticoagulation used during ECMO support may have favorably addressed the underlying thrombotic microangiopathy that attends Covid-19 infection (24). Early recognition of an impaired heparin response and fast transition to the alternative agent bivalirudin may have further treated the thrombotic microangiopathy. Early use of pre filter, high flow CRRT did appear to lower inflammatory markers and helped to carefully adjust intravascular volume in a fashion that did not compromise ECMO blood flow. Attempts to use post filter replacement fluid were met with prohibitive levels of filter thrombosis despite regional and systemic anticoagulation. The degree of cytokine removal from the PrismaFlex CRRT machine with the HF1000 filter (Baxter Healthcare Corporation, Deerfield, IL) remains under investigation (25).

Five of the ECMO patients have received compassionate emergency use of convalescent plasma. All tolerated the convalescent plasma infusions well. Three of the five have been liberated from both ECMO and MV, while two remain on full ECMO and MV support. Further investigation is required to assess the efficacy of the convalescent plasma.

The effectiveness of prone positioning in Covid-19 severe ARDS patients prior to ECMO support led to an increase in prone positioning while on ECMO support. If patients failed to show any improvement after 72 hours of ECMO support, a trial of prone positioning was implemented. Patients were also mobilized into a vertical position using the specialty bed. The use of a newly FDA approved subcutaneous EEG may have enabled earlier detection and treatment of epileptiform activity and nonconvulsive seizures. The EEG criteria used to define seizure activity with this new device are evolving, but treatment of the patients' seizures improved their neurologic exams. Prolonged use of treatment dose anticoagulation after ECMO liberation may have mitigated some of the late thrombotic events described in prior Covid-19 populations.

The limitations of a small case series are clear. Any health systems outcomes are clearly influenced by preparation time and whether their beds are literally overflowing with critically ill patients. Our experience suggests a helpful role for extracorporeal support in select patients; however, the concerns of premature extrapolation of the data in a small series are readily appreciated.

Greater benefits are probable in a high-volume center with an experienced team that has time to prepare, and one that is not completely overwhelmed by a pandemic surge of patients. The rapid sharing of information from our colleagues in Asia, Europe, and the United States was extremely beneficial to those downstream in the pandemic, as care evolved dynamically as new experiences were reported.

Conclusion: In carefully selected patients at a well-resourced, high-volume center with an experienced team, ECMO may be beneficial in patients with Covid-19 severe respiratory failure. Efficacy of EMCO support is greater when applied as soon as optimal evidence-based interventions are unable to support the most critically ill Covid-19 patients.

\section{Author contributions:}

Manuscript preparation: EO, SM, AS, MD, TP. Manuscript revision: PTF, LA, JS, CK, HD, PM. Manuscript analysis: RS, ES, DT, ML, JC, JG, KL, MR, JW, KP, SV, LR

\section{References:}

1. Alshahrani MS, Sindi A., Alshamsi F, et al. Extracorporeal membrane oxygenation for severe Middle East respiratory syndrome coronavirus. Ann. Intensive Care. 2018;8.

2. Henry B. COVID-19, ECMO, and lymphopenia: a word of caution. Lancet Respir. Med. 2020.

3. Sultan I, Habertheurer A, et al. The role of extracorporeal life support for patients with COVID-19: Preliminary results from a statewide experience.J Card Surg. 2020 Apr 25. doi: 10.1111/jocs.14583.

4. Ruan Q, Yang K, Wang W, et.al. Clinical predictors of mortality due to COVID-19 based on an analysis of data of 150 patients from Wuhan. China Intensive Care Med. 2020.

5. Yang X, Yu Y, Xu J, et.al. Clinical course and outcomes of critically ill patients with SARS-CoV2 pneumonia in Wuhan, China: a single-centered, retrospective, observational study. Lancet Respir. 
Med. 2020;0.

6. Zhou F, Yu T, Du R, et.al. Clinical course and risk factors for mortality of adult inpatients with COVID-19 in Wuhan, China: a retrospective cohort study. Lancet. 2020.

7. 6. Wu C, Chen X, Cai Y, et.al. Risk factors associated with acute respiratory distress syndrome and death in patients with coronavirus disease 2019 pneumonia in Wuhan. China JAMA Intern Med. 2020.

8. Brandon MH, Lippi G. Poor survival with extracorporeal membrane oxygenation in acute respiratory distress syndrome (ARDS) due to coronavirus disease 2019 (COVID-19): Pooled analysis of early reports J Crit Care. 2020 Apr 1. doi: 10.1016/j.jcrc.2020.03.011 [Epub ahead of print]

9. MacLaren G, Fisher D, Brodie D. Preparing for the Most Critically Ill Patients With COVID-19The Potential Role of Extracorporeal Membrane Oxygenation. JAMA. 2020;323(13):1245-1246.

10. Gattinoni L, Coppola S, Cressoni M, et al. Covid-19 Does Not Lead to a "Typical" Acute Respiratory Distress Syndrome. AJRCCM Articles in Press. Published March 30, 2020 as 10.1164/rccm.2020030817LE.

11. Magro C, Mulvey JJ, Berlin D, et al. Complement associated microvascular injury and thrombosis in the pathogenesis of severe COVID-19 infection: a report of five cases. Translational Research. 2020; 000:1-13.https://doi.org/10.1016/j.trsl.2020.04.007

12. Helms $\mathrm{J}$ et al. High risk of thrombosis in patients in severe SARS-CoV-2 infection: a multicenter prospective cohort study. Intensive Care Medicine (2020); DOI: 10.1007/s00134-020-06062-x

13. Honore P, Hoste E, et al. Cytokine removal in human septic shock; Where are we and where are we going? Ann. Intensive Care. (2019) 9:56.

14. Al-Bawardy, et al. Extracorporeal membrane oxygenation in acute massive pulmonary embolism: a case series and review of the literature. Perfusion. 2019. Vol 34(1) 22-28.

15. Li, Y-C, Bai, W-Z, Hashikawa, T. The neuroinvasive potential of SARS-CoV2 may play a role in the respiratory failure of COVID-19 patients. J Med Virol. 2020;1-4.

16. Mao L, Jin H, Wang M, et al. Neurologic manifestations of hospitalized patients with coronavirus disease 2019 inWuhan, China. JAMA Neurol. Published online April 10, 2020. doi:10.1001/jamaneurol.2020.1127

17. Xie A, Lo P, Yan TD, Forrest P. Neurologic Complications of Extracorporeal Membrane Oxygenation: A Review. J Cardiothorac Vasc Anesth. 2017 Oct;31(5):1836-1846.

18. Sutter R, Tisljar K, Marsch S. Acute Neurologic Complications During Extracorporeal Membrane Oxygenation: A Systematic Review. Crit Care Med. 2018 Sep;46(9):1506-1513.

19. Hick J, Biddinger P, Novel Coronavirus and Old Lessons - Preparing the Health System for the Pandemic. March 25, 2020. N Engl J Med. DOI:10.1056/NEJMp20005118

20. Cerebral Performance Category scale. Adapted from: Grenvik A, Safar P, editors. Brain failure and resuscitation. New York: Churchill Livingstone; 1981; p.155-84

21. Emanuel E, Persad J. Fair Allocation of Scarce Medical Resources in the Time of Covid-19. March 23, 2020. N Engl J Med. DOI:10.1056/NEJMsb2005114

22. Schmidt M, Bailey M, Sheldrake J. Predicting Survival after extracorporeal membrane oxygenation for severe acute respiratory failure. The Respiratory Extracorporeal Membrane Oxygenation Surival Prediction (RESP) score. Am J Respir Crit Care Med. 2014 Jun 1;189(11):1374-82

23. Combes A, Hajage D, Capellier G, et al. Extracorporeal membrane oxygenation for severe acute respiratory distress syndrome. N Engl J Med 2018; 378:1965-1975

24. Connors JM, Levy JH, COVID-19 and its implications for thrombosis and anticoagulation. Blood. 2020 Apr 27. Pii: blood.2020006000.doi [Epub ahead of print]

25. Ongoing investigation: Osborn E, Preston T, Desai M, Moran P, et al. Cytokine Assessment, Attenuation and Clearance Through High-Volume Continuous Renal Replacement Therapy in Patients with Covid-19 Undergoing Mechanical Ventilation and Concurrently Supported with Extracorporeal Membrane Oxygenation (ECMO)

\section{Figures and Tables}

Table 1 


\section{Characteristic}

Admission Location

Home

Skilled Nursing Facility

Hospital Transfer

Age

Sex

Male

Female

Race

Caucasian

Black

Hispanic

Asian

Other

Body Mass Index (BMI)

Coexisting Disorder

Asthma

Hypertension

Chronic Kidney Disease

Current or former smoker

Chronic Obstructive Pulmonary Disease

Diabetes mellitus

Obstructive Sleep Apnea

New York Heart Association Class

Class I

Class II

Class III

Class IV
Patients

$\mathrm{N}=15$

$11(73 \%)$

0

$4(27 \%)$

$* 44 \pm 10$

$11(73 \%)$

$4(27 \%)$

0

$2(13 \%)$

$9(60 \%)$

$2(13 \%)$

$2(13 \%)$

*33 \pm 8.5

0

$8(53 \%)$

0

$3(20 \%)$

0

$8(53 \%)$

0

$15(100 \%)$

0

0

0

$* \pm$ values are mean \pm SD

Percentages may not total $100 \%$ due to rounding

Table 2 
Days symptomatic

Days on mechanical ventilatory support

$\mathrm{PaO} 2 / \mathrm{FiO} 2$ Ratio

Peak plateau pressure

Tidal volume $\leq 6 \mathrm{ml} / \mathrm{kg}$

No

Yes

Paralytic

Prone position

Inhaled pulmonary vasodilator use

Epoprostenol

Nitric oxide

Arterial blood gas

$\mathrm{pH}$

Carbon dioxide

Vasopressor use

Acute kidney injury

Fluid balance (liters)

$*$ values are mean \pm SD
* $12.7 \pm 5.8$

$* 4.5 \pm 2.7$

$* 99.4 \pm 31.6$

*30.2 \pm 3.8

$3(20 \%)$

$12(80 \%)$

$14(93 \%)$

$14(93 \%)$

$8(53 \%)$

$6(40 \%)$

$* 7.28 \pm 0.06$

*64. $3 \pm 17.8$

$8(53 \%)$

$9(60 \%)$

1.33 ( Range -1.5 to +5.9 )

Table 3

\begin{tabular}{|l|c|c|}
\hline Characteristic & Heparin $(\mathbf{u} / \mathrm{Kg} / \mathrm{Hr})$ & Bivalirudin $(\mathrm{mg} / \mathrm{Kg} / \mathrm{Hr})$ \\
\hline Starting dose & $13 \pm 4$ & 0.19 \\
\hline Titration Goal & & 0 \\
\hline Moderate intensity & 7 & 7 \\
\hline High intensity & 1 & 4 \\
\hline Other & 3 & $85 \pm 18$ \\
\hline Average aPTT (seconds) & $73 \pm 23$ & $0.27 \pm 0.11$ \\
\hline Therapeutic Dose* & $17 \pm 5$ & $17 \pm 17$ \\
\hline Hours to therapeutic & $16 \pm 9$ & $17 \pm 16$ \\
\hline TEG R (seconds) & $20 \pm 12$ & -- \\
\hline Changed to bivalirudin & 8 & \\
\hline
\end{tabular}

- Moderate intensity = goal aPTT 70-90 seconds, High intensity= goal aPTT 75-95 seconds, Other $=$ titration not per protocol.

*Therapeutic dose not achieved in two patients

Table 4 


\begin{tabular}{|c|c|c|c|c|c|}
\hline Patient & $\begin{array}{l}\text { Fluid balance } \\
\text { prior to CRRT } \\
\text { (L) }\end{array}$ & $\begin{array}{c}\text { Highest } \\
\text { replacement } \\
\text { flow rate } \\
(\mathrm{mL} / \mathrm{Kg} / \mathrm{Hr})\end{array}$ & $\begin{array}{l}\text { Duration of } \\
\text { CRRT (Days) }\end{array}$ & $\begin{array}{c}\text { Average } \\
\text { Replacement } \\
\text { flow rate } \\
(\mathrm{mL} / \mathrm{Kg} / \mathrm{Hr})\end{array}$ & Status \\
\hline 1 & 5.8 & 62 & 9 & 42 & Recovery \\
\hline 2 & 2.6 & 25 & 7 & 25 & Recovery \\
\hline 3 & 3.5 & 60 & 6 & 31 & Recovery \\
\hline 4 & 5.9 & 24 & 6 & 24 & Recovery \\
\hline 5 & -- & -- & -- & -- & -- \\
\hline 6 & 1.39 & 46 & 7 & 26 & Current CRRT \\
\hline 7 & 0.28 & 26 & 6 & 25 & Recovery \\
\hline 8 & -- & -- & -- & -- & -- \\
\hline 9 & 3.5 & 31 & 27 & 19 & Current CRRT \\
\hline 10 & -- & -- & -- & -- & -- \\
\hline 11 & 1.97 & 76 & 11 & 53 & Hemodialysis \\
\hline 12 & 0.5 & 38 & 7 & 38 & Recovery \\
\hline 13 & 2.44 & 51 & 8 & 24 & Current CRRT \\
\hline 14 & -1.45 & 31 & 5 & 25 & Recovery \\
\hline 15 & 1.02 & 43 & 6 & 33 & Current CRRT \\
\hline
\end{tabular}

*CRRT - Continuous renal replacement therapy patients with no data did not require CRRT

Patients without data did not receive or require CRRT

Recovery- No ongoing dialysis requirement

Current CRRT-currently receiving CRRT at the time of publication

Hemodialysis- currently receiving hemodialysis

Table 5 
Complication

Thrombotic Event*

Cannula thrombosis

Early oxygenator thrombus

Emergent circuit exchange

CRRT filter thrombus, multiple

Pulmonary embolus

Post ECMO DVT

Heparin resistance

Type 2 HIT

Hemorrhage

Secondary infection

Pneumothorax

Right ventricular failure •

Neurologic complications

Epileptiform discharge

Autonomic dysfunction

Critical illness polyneuropathy

Encephalopathy

Cerebral ischemia or hemorrhage
Rate

Number of patients and (\%)

$10(66 \%)$

$2(13 \%)$

$5(33 \%)$

$4(27 \%)$

$4(27 \%)$

$1(7 \%)$

$2(13 \%)$

$8(53 \%)$

$1(7 \%)$

$4(27 \%)$

$8(53 \%)$

$2(13 \%)$

$3(20 \%)$

$6(40 \%)$

$7(46 \%)$

$6(40 \%)$

$6(40 \%)$

0

*Thrombotic event are defined by the components listed below the thrombotic event

- Hemorrhage defined as greater than one unit of PRBC over a 24 hour period

-Right ventricular failure is defined by impaired perfusion and ECHO findings

Table 6

CPC 1 Normal life, independent, mild disability

CPC 2 Disabled but independent enough for part time work

CPC 3 Conscious but disabled and dependent

CPC 4 Unconscious; coma or vegetative state

CPC 5 Brain death or dead by traditional criteria

Adapted from: Grenvik A, SafarP, editors. Brain failure and resuscitation. New

York: Churchill Livingstone; $1981 ;$ p. 155-84

Figure 1 


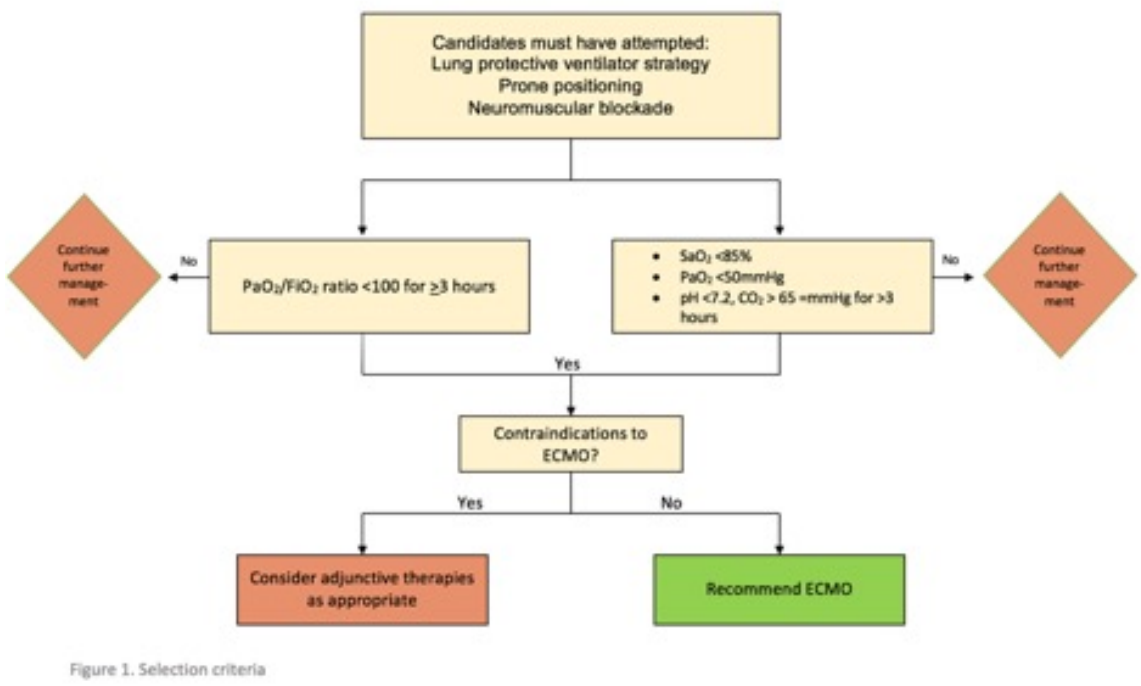

Figure 2 


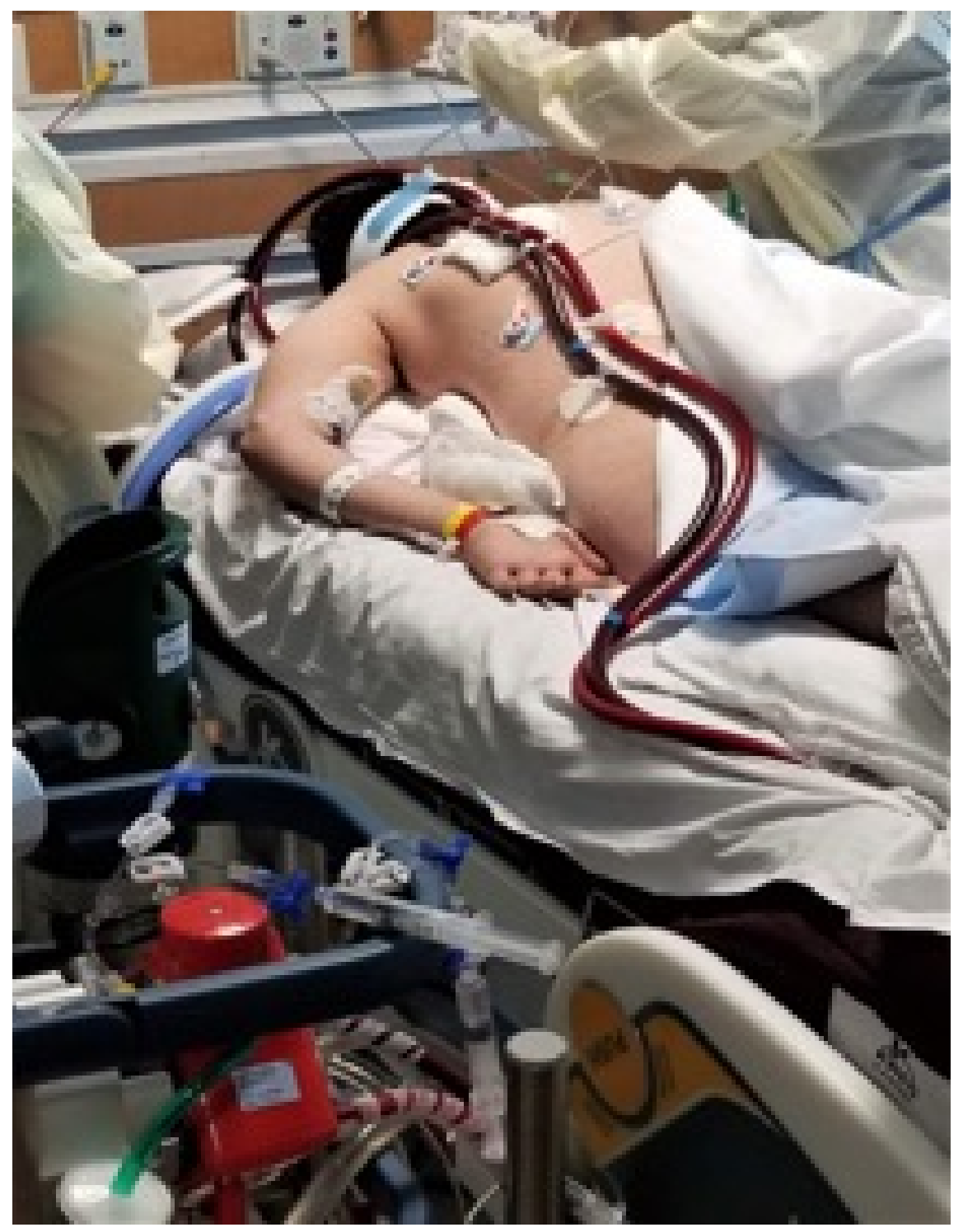

Figure 2. Prone position during ECMO

Figure 3 


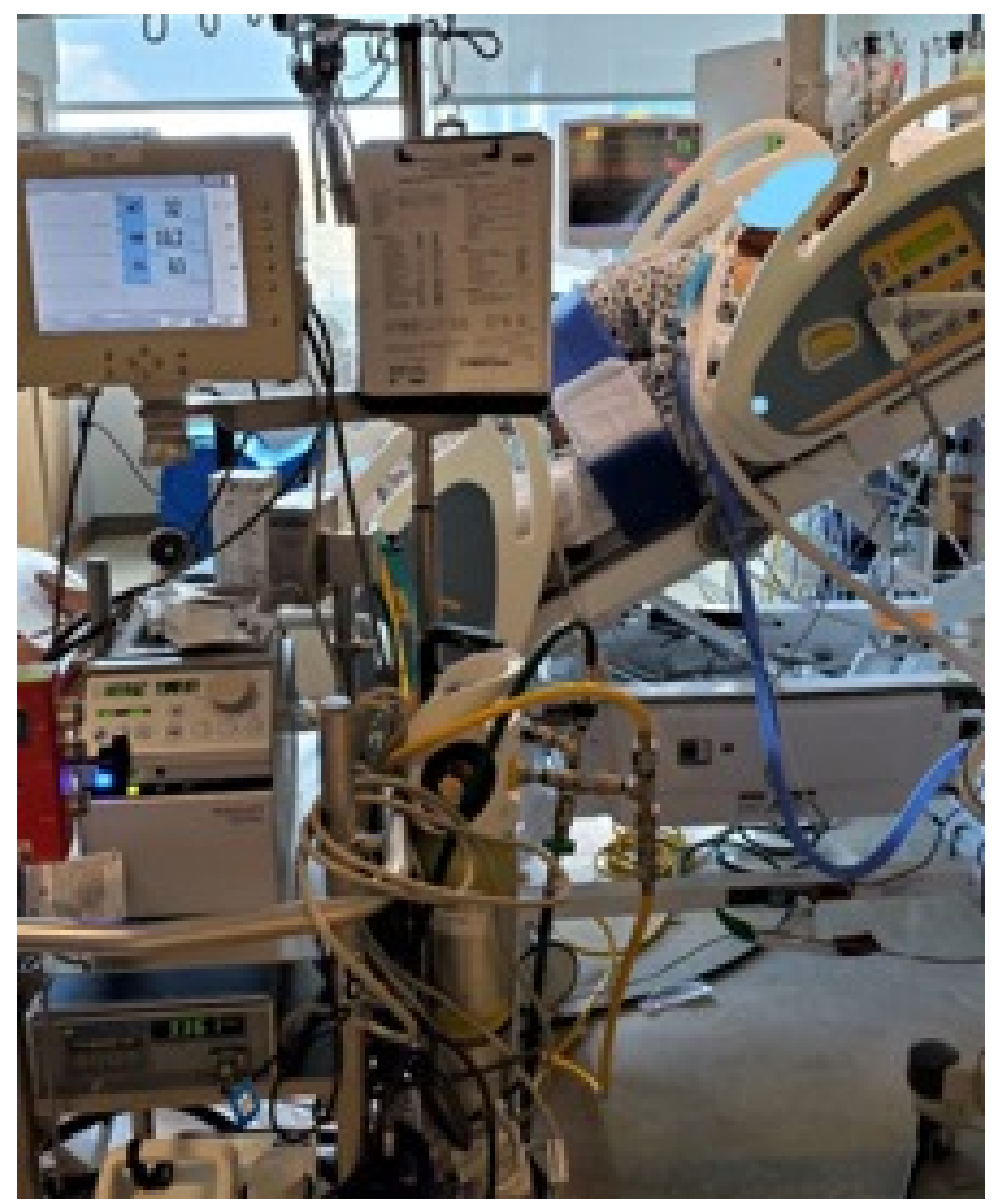

Figure 3. Verticalization using special bed

Figure 4 


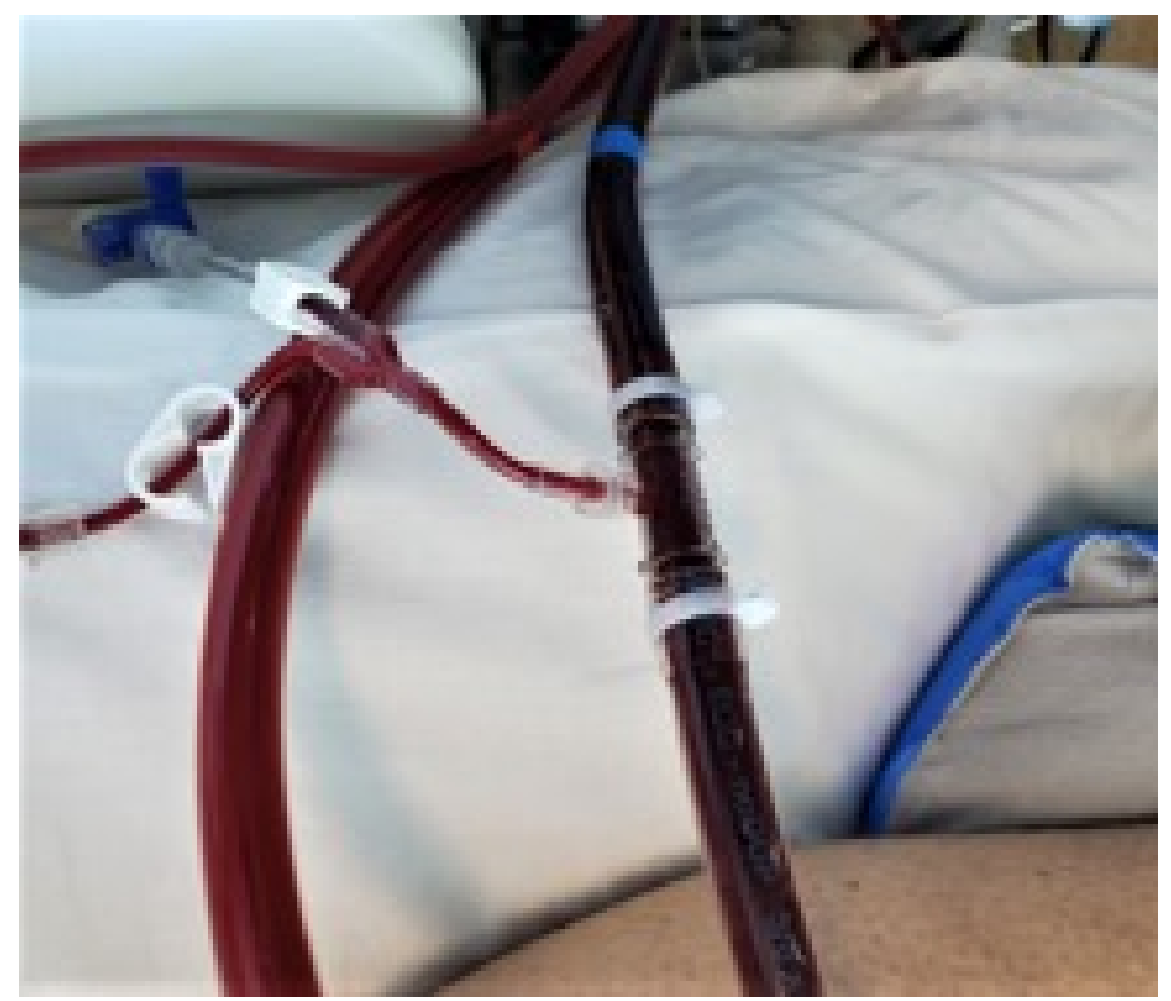

Figure 4: Access into venous return line of ECMO circuit to facilitate high flow rates during CRRT.

\section{Figure 5}

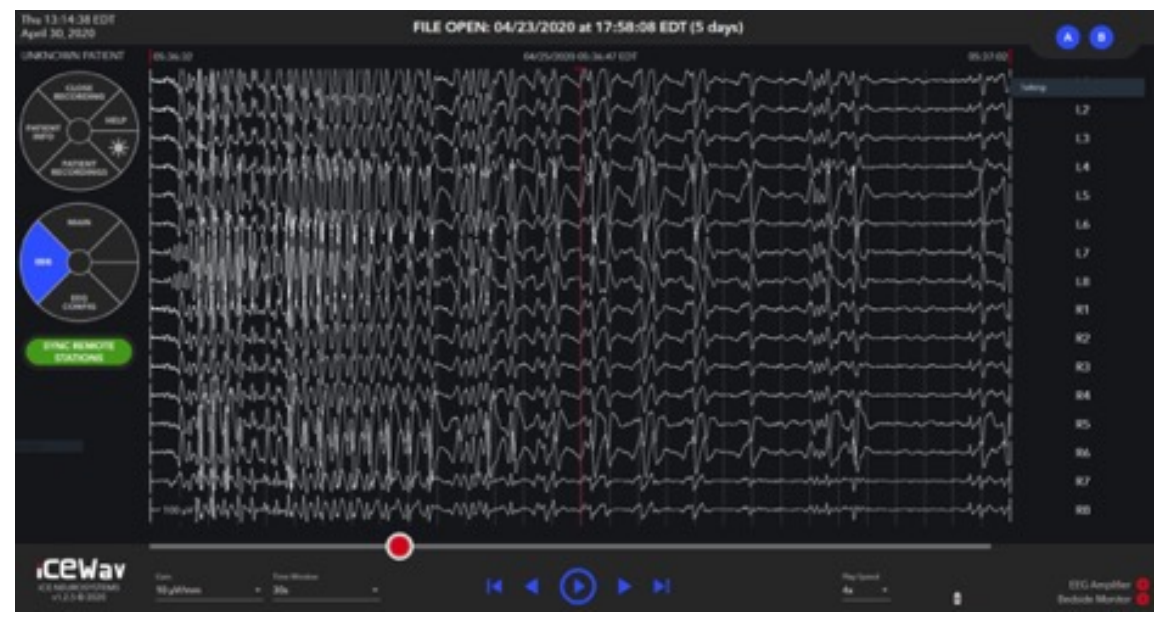

Figure 5. Bilateral generalized epileptiform discharges recorded from 2 subcutaneous parasagittal 10-contact electrodes (Ad-Tech Medical, Racine WI) and displayed with iCEWav software (Ice Neurosystems, Washington DC).

\section{Figure 6}




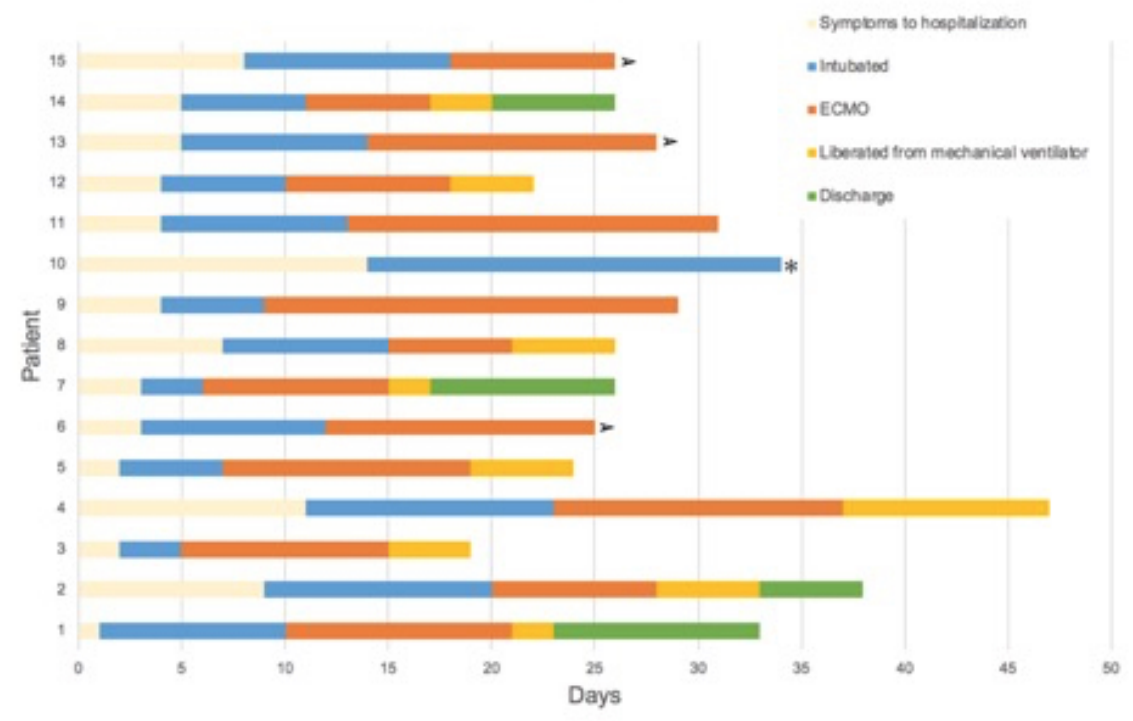

* Patient expired

> On ECMO at time of publication

Figure 7

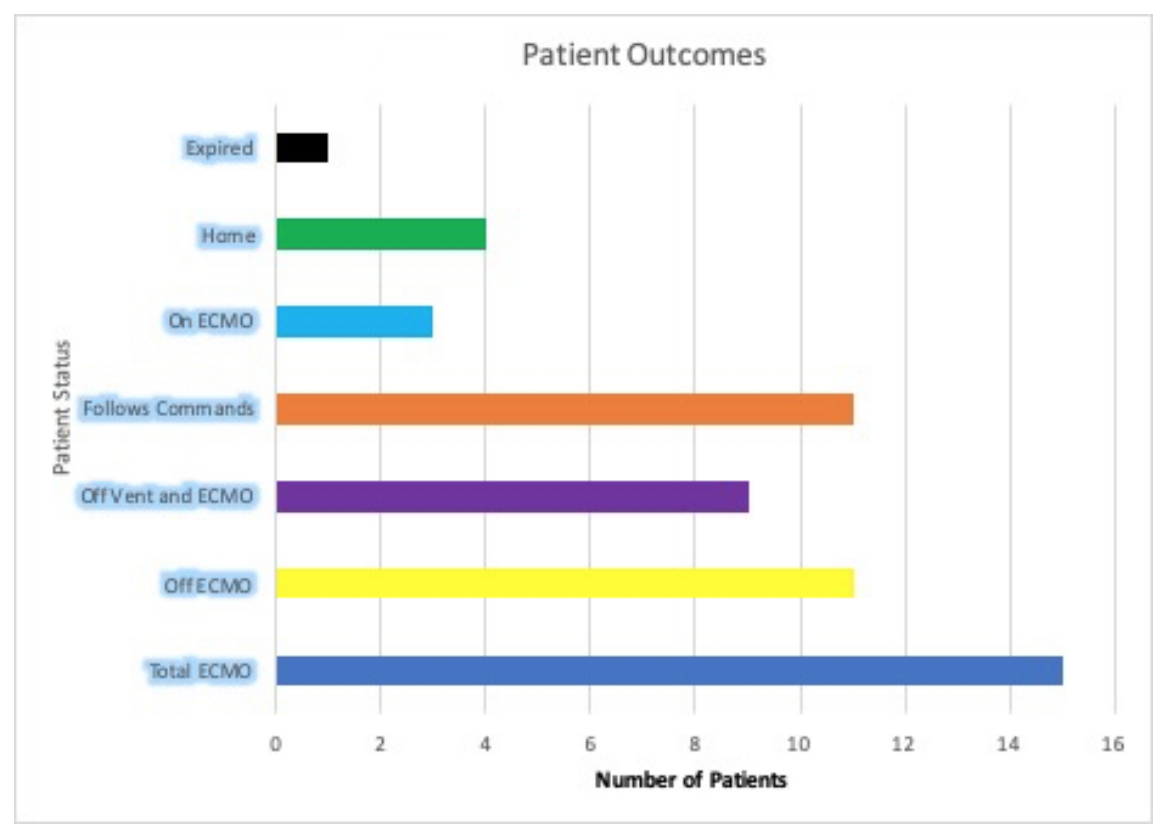

\section{Legends}

Figure 1. Selection criteria for VV ECMO

Figure 2. ECMO patient in prone position

Figure 3. Verticalization of the ECMO patient in the specialty bed

Figure 4. Access into venous limb of the ECMO circuit 


\section{Figure 5. EEG Epileptiform Activity}

Bilateral generalized epileptiform discharges recorded from 2 subcutaneous parasagittal 10-contact electrodes (Ad-Tech Medical, Racine WI) and displayed with iCEWav software (Ice Neurosystems, Washington D.C.).

Figure 6. Hospital Course

Figure 7. Patient Outcomes

Table 1. Clinical characteristics of the patients at baseline

Table 2. Pre-Extracorporeal Membrane Oxygenation characteristics

Table 3. Anticoagulation table

Table 4. Continuous Renal Replacement Therapy

Table 5. Complications

Table 6. Cerebral Performance Category 